\title{
Testicular tuberculosis: A case report and literature review
}

\section{Tuberculosis testicular, presentación de un caso y revisión de la literatura}

\author{
iDEnrique de Jesús Cerda-Guerrero, ${ }^{* *}$ iD José Arturo Rodríguez-Rivera. ${ }^{1}$
}

Keywords:

Testicular tuberculosis, genital tuberculosis, genitourinary tuberculosis.

Correspondencia:

*Autor de correspondencia: Enrique Cerda-Guerrero. Av Zoquipan 1050, Zoquipan, 45170, Zapopan, Jalisco. Correo electrónico: dr.quiquecerdauro@hotmail.com

\section{Abstract}

Clinical case: A 33-year-old man with a family history of pulmonary tuberculosis sought medical consultation due to predominantly nocturnal fever and the perception of a painless right testicular mass. He had no other associated symptoms and tumor markers were within normal ranges.

Relevance: According to World Health Organization figures, infection due to Mycobacterium tuberculosis is the most frequent opportunistic infection worldwide.

Conclusion: The characteristic clinical presentation of testicular tuberculosis is a painless testicular mass that is difficult to differentiate from a malignant tumor. Nonspecific laboratory and imaging findings, together with low diagnostic suspicion, result in a high percentage of late diagnoses. At present, the disease is detected through pathologic findings in the surgical specimen, in a considerable number of patients. Even though short-term and medium-term outcomes tend to be favorable, one of the long-term complications of testicular tuberculosis is infertility, secondary to the destruction of the testicular parenchyma and/or obstruction of the sperm release pathway..

Citación: Cerda-Guerrero E.J., Rodríguez-Rivera J.A. Tuberculosis testicular, presentación de un caso y revisión de la literatura. Rev. Mex. Urol. 2020;80(3):pp 1-9

\footnotetext{
${ }^{1}$ Secretaría de Salud, Hospital General de Occidente, Zapopan, México.
}

Recepción: 18 de diciembre de 2019

Aceptación: 25 de abril de 2020 


\section{Tuberculosis testicular, presentación de un caso y revisión de la literatura. Cerda-Guerrero E. de J., et al.}

Palabras clave:

Tuberculosis testicular, tuberculosis genital, tuberculosis genitourinaria

\section{Resumen}

Descripción del caso clínico: Presentamos el caso de un paciente masculino de 33 años con historia familiar de tuberculosis pulmonar, quien acude a consulta por presentar fiebre de predominio nocturno y percepción de masa testicular derecha no dolorosa, sin otro síntoma asociado, marcadores tumorales en rangos normales.

Relevancia: De acuerdo con cifras de la OMS (Organización Mundial de la Salud), la infección por Mycobacterium tuberculosis es considerada la infección oportunista más frecuente en el mundo.

Conclusión: La presentación clínica característica de la tuberculosis testicular (TbT) es una masa testicular no dolorosa, difícil de diferenciar de una tumoración maligna. Los hallazgos inespecíficos en los exámenes de laboratorio e imagen acompañados de baja sospecha diagnóstica llevan a un alto porcentaje de diagnósticos tardíos. Hoy en día una cantidad considerable de pacientes se detectan por hallazgos de patología en la pieza quirúrgica. Si bien el pronóstico a corto y mediano plazo suele ser favorable, una de las complicaciones a largo plazo de la TbT es la infertilidad secundaria a la destrucción del parénquima testicular y/o la obstrucción del trayecto de salida de los espermatozoides.

\section{Antecedentes}

La OMS estima que la tercera parte de la población mundial se encuentra infectada por tuberculosis $(\mathrm{Tb})$, considerada la infección oportunista más común en pacientes inmunosuprimidos, y ha cobrado importancia en los últimos años. ${ }^{(1-3)}$ La presentación pulmonar es la forma inicial y más común de $\mathrm{Tb}$. La forma extrapulmonar (TbEP) se presenta en un 10$15 \%$ de los pacientes y de esta la tuberculosis genitourinaria (TbGU) representa el 15\% (una sexta parte de la TbEP). ${ }^{(2-3)}$ La TbGU afecta principalmente a hombres de 20-40 años de edad e involucra en orden de frecuencia al riñón, uréter, testículo, vejiga, próstata, uretra y pene. La tuberculosis genital aislada es rara y el principal sitio afectado es el epidídimo, seguido por las vesículas seminales, próstata, testículo y el conducto deferente en ese orden de frecuencia. ${ }^{(2-3)}$

\section{Caso clínico}

Paciente masculino de 33 años con antecedentes familiares de tuberculosis e historia personal de hacinamiento, acude a consulta por cuadro de dos meses de evolución con percepción de masa no dolorosa y aumento de volumen en testículo derecho. Al interrogatorio dirigido, refiere fiebre no cuantificada de predominio nocturno desde hace siete meses, niega la presencia de síntomas urinarios bajos. 
A la exploración física presenta aumento difuso de volumen en testículo derecho, no desencadenante de dolor, no se palpan lesiones focales ni otras anormalidades. El examen general de orina muestra piuria estéril y en el urocultivo no hay desarrollo de microorganismos. Se solicitan marcadores tumorales: subunidad beta gonadotropina coriónica, alfa fetoproteína y deshidrogenasa láctica, los cuales se encuentran en rangos normales. Se realiza ultrasonido Doppler testicular, encontrando aumento difuso de volumen en testículo derecho en compo- ración con teste contralateral, además de áreas hipoecoicas mal delimitadas en epidídimo derecho, a la aplicación del Doppler color no hay evidencia de aumento en la vascularidad (Figuras 1 y 2). Como parte del abordaje diagnóstico se realiza PPD, el cual se reporta positivo. Se inicia tratamiento antifímico con 4 fármacos (Isoniacida, Rifampicina, Pirazinamida y Etambutol). En el seguimiento, luego de 30 días con tratamiento antifímico, no se observa respuesta (sin cambios a la exploración física), por lo que se decide realizar orquiectomía derecha.

Figuras 1. Ultrasonido doppler testicular realizado con ultrasonido planar, se aprecia un aumento difuso del volumen testicular, destaca la presencia de lesiones hipoecoicas difusas poco delimitadas en el epidídimo derecho

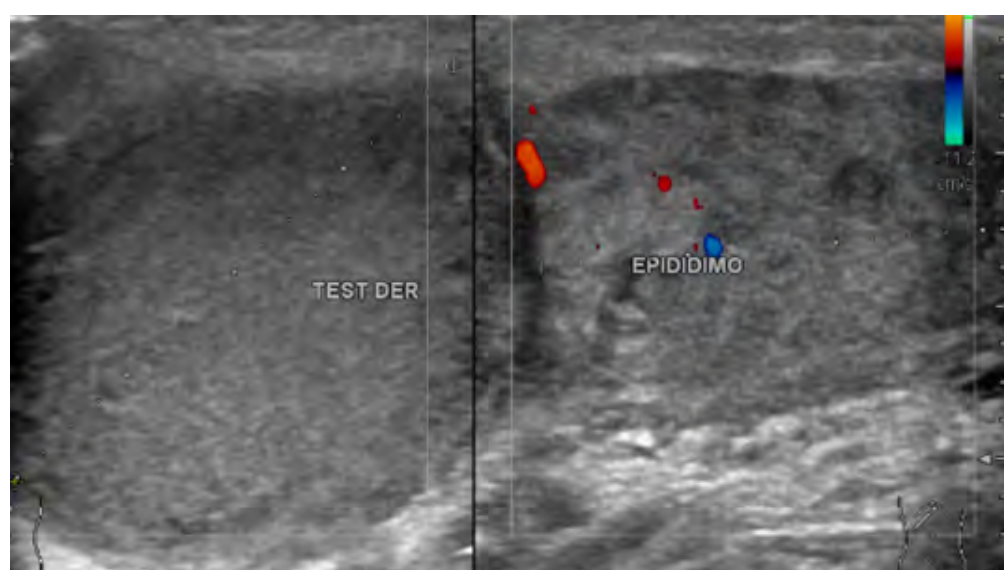

Figuras 2. Ultrasonido doppler testicular realizado con ultrasonido planar, se aprecia un aumento difuso del volumen testicular, destaca la presencia de lesiones hipoecoicas difusas poco delimitadas en el epidídimo derecho

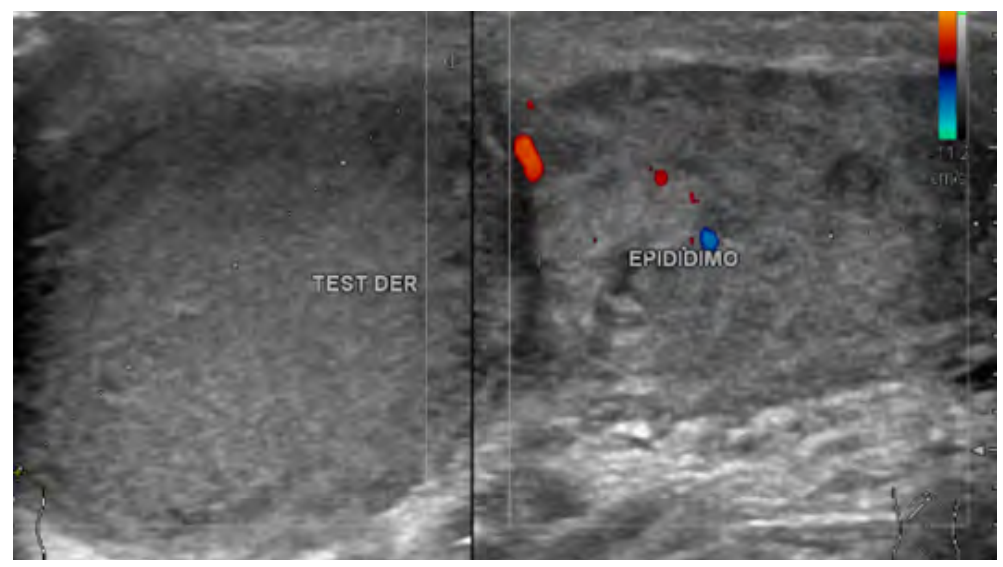




\section{Hallazgos en la Cirugía}

Se encuentra hidrocele de $20 \mathrm{cc}$ en bolsa escrotal derecha, lesión nodular exofítica en polo inferior de testículo derecho (Figuras 3 y 4), epidídimo engrosado sin lesiones focales, se realiza orquiectomía sin complicaciones.

Figuras 3. Imágenes transquirúrgicas, se muestra testículo derecho con lesión nodular exofítica en el polo inferior, epidídimo engrosado sin lesiones evidentes

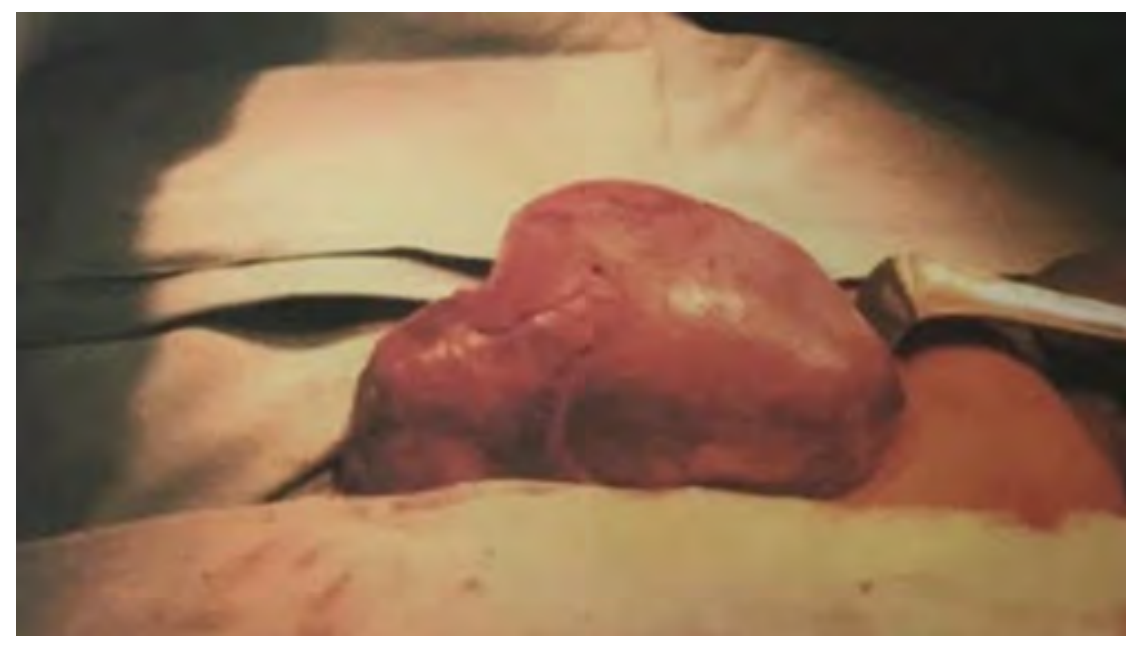

Figuras 4. Imágenes transquirúrgicas, se muestra testículo derecho con lesión nodular exofítica en el polo inferior, epidídimo engrosado sin lesiones evidentes

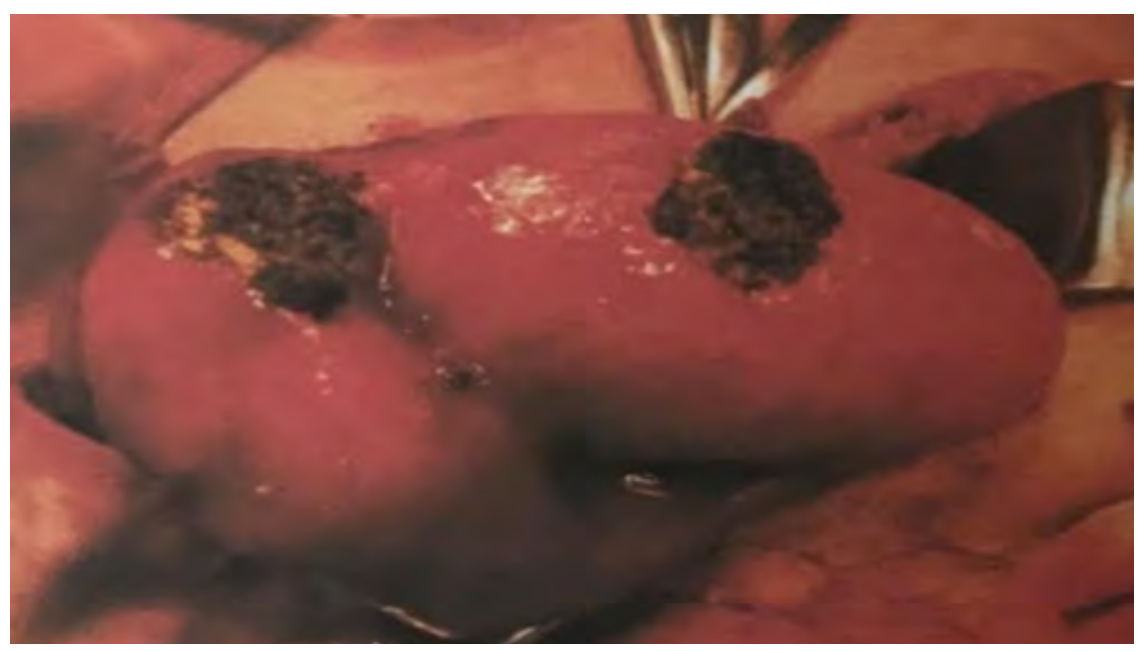

El reporte de patología describe inflamación crónica granulomatosa, células gigantes tipo Langhans y necrosis caseosa central (Figura 5). Con la tinción de Ziehl-Neelsen se identifican bacilos ácido alcohol resistentes (Figura 6), se completa ciclo de 6 meses de tratamiento, actualmente paciente asintomático. 
Figura 5. Corte histológico, con tinción de hematoxilina-eosina, se aprecia inflamación crónica granulomatosa, presencia de células gigantes tipo Langhans $y$ necrosis caseosa central

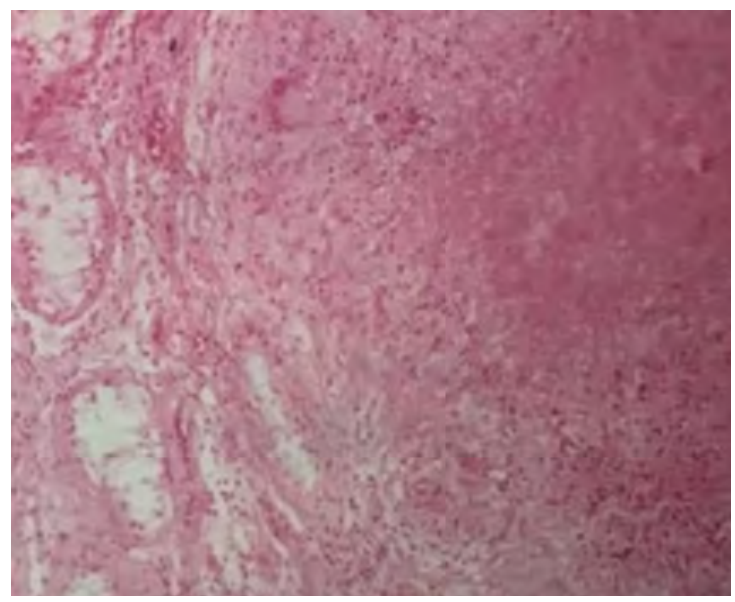

Figura 6. Tinción de Ziehl-Neelsen se identifican bacilos ácido alcohol resistentes

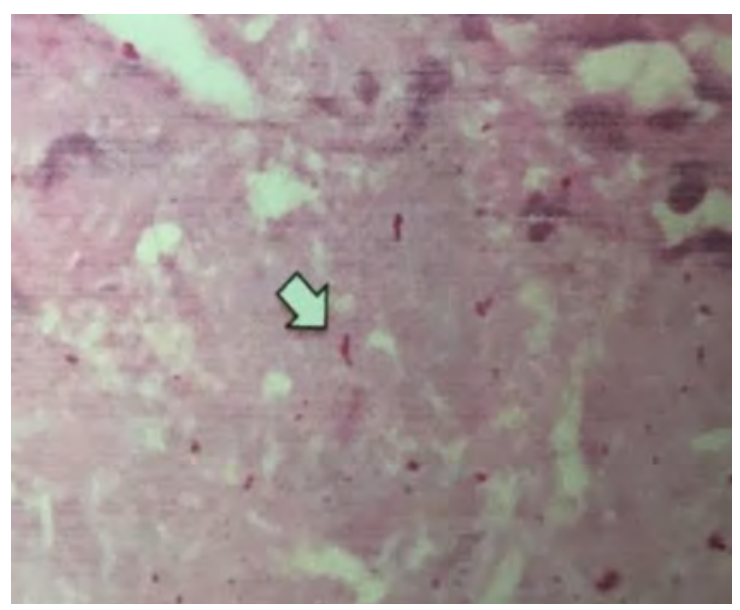

\section{Discusión}

La Tb sigue siendo una importante causa de morbimortalidad en países en vías de desarrollo, la OMS ha considerado que una tercera parte de la población mundial se encuentra infectada (más de 2 billones de personas), con una incidencia de 9 millones de casos nuevos por año reportados para 2007. ${ }^{(2-4)}$

La TbGU fue descrita por primera vez por Willbolz (1937) y comprende el 8-15\% de la Tb EP. ${ }^{(3,4)}$ La afección genital aislada se observa en un $28 \%$ de los pacientes con TbGU y muestra predilección por pacientes del sexo masculino de 30 a 50 años. ${ }^{(4,5)}$ En hombres con Tb genital el sitio de afección más común es el epidídimo, seguido por las vesículas seminales, próstata, testículo y el conducto deferente. ${ }^{(3)}$

El mecanismo de diseminación del bacilo de la $\mathrm{Tb}$ hasta el saco escrotal es controversial, se considera que la vía más probable de acceso es la diseminación retrograda desde un tracto urinario infectado hacia la próstata, seguido por la diseminación canalicular a las vesículas seminales, conducto deferente y epidídimo. Otros mecanismos de acceso considerados son la vía hematógena y linfática, algunos autores consideran a la vía hematógena como la principal forma de diseminación. ${ }^{(4-6)}$ La transmisión por vía sexual es posible, aunque poco frecuente y se sustenta por la identificación de micobacterias en muestras de semen de pacientes con prostatitis y la descripción anecdótica de unos cuantos casos reportados en la literatura. (4) La infección suele diseminarse desde el epidídimo, una vez en el testículo esta aparece por primera vez en el polo inferior, debido al mayor aporte sanguíneo en esta zona. ${ }^{(4-6)}$

\section{Cuadro clínico}

La TbT se suele presentar como una masa no dolorosa, difícil de distinguir a la palpación de una tumoración maligna, algunos de los síntomas que se presentan con mayor frecuencia 


\section{Tuberculosis testicular, presentación de un caso y revisión de la literatura. Cerda-Guerrero E. de J., et al.}

son: fiebre, malestar general y pérdida de peso. $(1,4,7)$ La hematuria y la piuria estéril son los hallazgos clásicos de la tuberculosis genitourinaria, sin embargo, para que estos se presenten, se requiere de la afección significativa en otro sitio del tracto genitourinario. ${ }^{(4)}$

\section{Diagnóstico}

El ultrasonido testicular es el estudio de imagen de elección para el abordaje inicial, Muttarak et al., clasificaron el compromiso testicular en cuatro tipos, dependiendo de los hallazgos ecosonográficos, estos son:

1. Aumento difuso de tamaño, heterogéneo e hipoecoico.

2. Aumento difuso de tamaño homogéneo e hipoecoico.

3. Crecimiento nodular heterogéneo e hipoecoico.

4. Miliar. $^{(8,9)}$

En el US la visualización de una lesión testicular con extensión al epidídimo orienta al diagnóstico de infección sobre el de malignidad, otros hallazgos ecosonográficos inespecíficos que sugieren TbT son el crecimiento heterogéneo con lesiones hipoecoicas en el epidídimo. ${ }^{(2-8)}$
El US Doppler color testicular ayuda a diferenciar la TbT de la torsión testicular, en esta ultima el flujo sanguíneo al testículo se encuentra disminuido o ausente, mientras que en pacientes con inflamación testicular el flujo se encuentra aumentado. ${ }^{(2-4)}$ El diagnóstico definitivo de TbT se realiza con la identificación del Mycobacterium tuberculosis en la orina, sin embargo, los cultivos de orina para tuberculosis usualmente resultan negativos. ${ }^{(10-12)}$

La citología obtenida por aspiración con aguja fina muestra imágenes características en la tinción Ziehl-Neelsen que incluyen la presencia de un granuloma epitelioide con halo necrótico, además de la identificación de bacilos rectos.

El PPD o prueba de tuberculina es una herramienta útil en el diagnóstico, sin embargo, requiere de personal capacitado para su interpretación. ${ }^{(2-7-11)}$ El uso de detección de PCR para Tb ha incrementado la sensibilidad y especificidad en el diagnóstico de tuberculosis a un $96 \%$ y $98 \%$ respectivamente. (5-6-11) $^{-1}$

Dentro de los diagnósticos diferenciales de la TbT se encuentran: tumor testicular malígno, infarto testicular, orquitis aguda y otras afecciones granulomatosas menos frecuentes.

En el cuadro 1 se describe el abordaje diagnóstico y terapéutico del paciente masculino con sospecha de tuberculosis geneitourinaria. ${ }^{(13)}$ 
Cuadro 1. Algoritmo de manejo y tratamiento de la tuberculosis testicular ${ }^{(13)}$

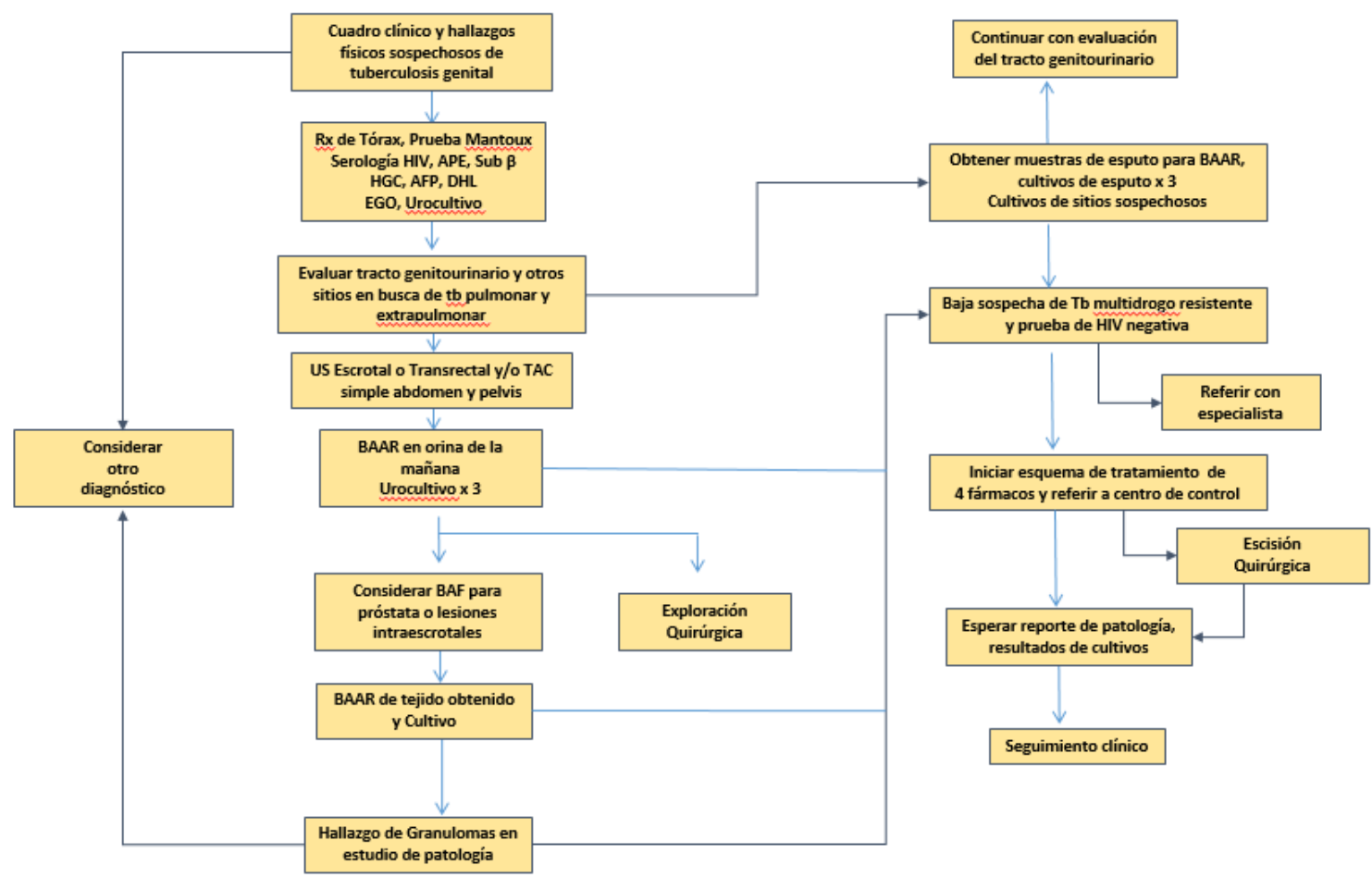

\section{Tratamiento}

De acuerdo con la NOM-006-SSA2-2013, el tratamiento de elección consiste en un esquema de 4 antifimicos durante 6 meses (25 semanas), hasta completar 105 dosis y se divide en 2 etapas:

\section{Fase Intensiva (60 dosis)}

Diario, de lunes a sábado: HRZE (Isoniazida, Rifampcina, Pirazinamida y Etambutol).

\section{Fase de Sostén (45 dosis)}

Intermitente 3 veces a la semana con HR (Isoniazida y Rifampicina). ${ }^{(14)}$

El manejo quirúrgico se debe reservar para los casos que no presentan respuesta rápida y adecuada o en aquellos en los que la sospecha de malignidad es alta. ${ }^{(3-14)}$

\section{Pronóstico}

Con el diagnóstico y tratamiento oportuno, la mortalidad asociada a la TbT es baja. En cuanto a las complicaciones a largo plazo, la principal y más preocupante es la infertilidad, la cual ocurre por la obstrucción del flujo espermático o por la destrucción del parénquima testicular. ${ }^{(2,4,15)}$

\section{Conclusión}

A pesar de tratarse de una enfermedad poco frecuente, el índice alto de sospecha en pacien- 
tes en quienes no exista duda razonable de malignidad evitará la realización temprana de una orquiectomía que pudiera resultar en sobretratamiento, en especial en pacientes con antecedente de tuberculosis o con factores de riesgo para esta. Lamentablemente debido principalmente a las caracteristícas de la enfermedad y a la situación sanitaria actual en nuestro país, en la mayoría de los pacientes la detección de la tuberculosis testicular se realiza posterior a la orquiectomía.

\section{Referencias}

1. Sanchez Lopez HM. Manual Practico de Urologia. Cuellar; 2014. [accessed 14 May 2020] Available from: http://www.cuellarayala.com/ shop/manual-practico-de-urologia/

2. Ghoneim IA, Rabets JC, Mawhorter SD. Chapter 16: tuberculosis and other opportunistic infections of the genitourinary system. Campbell-Walsh Urology 10 th Edition Editors: Wein AJ, Kavoussi LR, Partin AW, Novick AC, Peters CA Philadelphia. 2012;1:471-4.

3. Das A, Batabyal S, Bhattacharjee S, Sengupta A. A rare case of isolated testicular tuberculosis and review of literature. J Family Med Prim Care. 2016;5(2):468-70. doi: https://dx.doi. org/10.4103\%2F2249-4863.192334

4. Jacob JT, Nguyen TML, Ray SM. Male genital tuberculosis. Lancet Infect Dis. 2008 May;8(5):335-42. doi: https://doi. org/10.1016/S1473-3099(08)70101-4

5. Abraham S, Izaguirre Anariba DE, Dua K, Mir M, Ankireddypalli A. A case of testicular tuberculosis mimicking malignancy in a healthy young man. Therapeutic Advances in Infection. 2016 Jun 1;3(3-4):110-3. doi: $10.1177 / 2049936116655261$
6. Cho YS, Joo KJ, Kwon CH, Park HJ. Tuberculosis of testis and prostate that mimicked testicular cancer in young male soccer player. J Exerc Rehabil. 2013 Jun 30;9(3):389-93. doi: https:// dx.doi.org/10.12965\%2Fjer.130046

7. Badmos K. Tuberculous epididymo-orchitis mimicking a testicular tumour: a case report. Afr Health Sci. 2012 Sep;12(3):395-7. doi: https://dx.doi.org/10.4314\%2Fahs.v12i3.26

8. Muttarak M, Peh WC, Lojanapiwat B, Chaiwun B. Tuberculous epididymitis and epididymoorchitis: sonographic appearances. AJR Am J Roentgenol. 2001 Jun;176(6):1459-66. doi: https://doi.org/10.2214/ajr.176.6.1761459

9. Ogbole GI, Bassey OS, Okolo CA, Ukperi SO, Ogunseyinde AO. Testicular tuberculosis presenting with metastatic intracranial tuberculomas only: a case report. J Med Case Reports. 2011 Mar 13;5:100. doi: https://dx.doi. org/10.1186\%2F1752-1947-5-100

10. Chirindel A, Martinez F, Gagliardi JA, Armm MF. Testicular Tuberculosis Without Epididymitis Simulating Neoplasm. Radiol Case Rep. 2015 Nov 6;3(3). [accessed 14 May 2020] Available from: https://www.ncbi.nlm.nih.gov/ pmc/articles/PMC4897020/

11. Brooks GF, Morse SA, Butel JS. Microbiología Médica de Jawetz, Melnick y Adelberg 17a. Ed El Manual Moderno, SA de CV México DF. 2002;

12. Shugaba AI, Rabiu AM, Uzokwe C, Matthew RM. Tuberculosis of the Testis: A case Report. Clin Med Insights Case Rep. 2012 Jan 1;5:CCRep. S9451. doi: https://doi.org/10.4137\%2FCCRep. S9451

13. Cek M, Lenk S, Naber KG, Bishop MC, Johansen TEB, Botto $\mathbf{H}$, et al. EAU guidelines for the management of genitourinary tuberculosis. Eur Urol. 2005 Sep;48(3):353-62. doi: https://doi. org/10.1016/j.eururo.2005.03.008 
14. NORMA Oficial Mexicana NOM-006SSA2-2013, Para la prevención y control de la tuberculosis. 2013. [accessed 14 May 2020] Available from: http://dof.gob.mx/nota_detalle. php? codigo $=5321934 \&$ fecha $=13 / 11 / 2013$

15. Kinnear N, Hoh I, Campillo P, Bolt J. Tuberculous epididymo-orchitis masquerading as acute scrotum. BMJ Case Rep. 2016 Feb 4;2016. [accessed 14 May 2020] Available from: https://www.ncbi.nlm.nih.gov/pmc/articles/ PMC4746547/ 
Tuberculosis testicular, presentación de un caso y revisión de la literatura. Cerda-Guerrero E. de J., et al. 
Tuberculosis testicular, presentación de un caso y revisión de la literatura. Cerda-Guerrero E. de J., et al. 
Tuberculosis testicular, presentación de un caso y revisión de la literatura. Cerda-Guerrero E. de J., et al. 
Tuberculosis testicular, presentación de un caso y revisión de la literatura. Cerda-Guerrero E. de J., et al. 